PROCEEDINGS OF THE

AMERICAN MATHEMATICAL SOCIETY

Volume 134, Number 8, Pages 2285-2294

S 0002-9939(06)08238-4

Article electronically published on February 2, 2006

\title{
ON THE DERIVATIVES OF THE BEREZIN TRANSFORM
}

\author{
MIROSLAV ENGLIŠ AND GENKAI ZHANG
}

(Communicated by Joseph A. Ball)

\begin{abstract}
Improving upon a recent result of L. Coburn and J. Xia, we show that for any bounded linear operator $T$ on the Segal-Bargmann space, the Berezin transform of $T$ is a function whose partial derivatives of all orders are bounded. Similarly, if $T$ is a bounded operator on any one of the usual weighted Bergman spaces on a bounded symmetric domain, then the appropriately defined "invariant derivatives" of any order of the Berezin transform of $T$ are bounded. Further generalizations are also discussed.
\end{abstract}

\section{INTRODUCTION}

Consider the Segal-Bargmann (or Fock) space $A^{2}\left(\mathbf{C}^{n}, d \mu\right)$ of all entire functions in $L^{2}\left(\mathbf{C}^{n}, d \mu\right)$, where $d \mu$ is the Gaussian measure

$$
d \mu(z)=(2 \pi)^{-n} e^{-\|z\|^{2} / 2} d v(z),
$$

$d v$ being the Lebesgue volume on $\mathbf{C}^{n}$. It is well known that $A^{2}\left(\mathbf{C}^{n}, d \mu\right)$ admits a reproducing kernel, namely, for any $x \in \mathbf{C}^{n}$,

$$
f(x)=\int_{\mathbf{C}^{n}} f(y) K(x, y) d \mu(y) \quad \forall f \in A^{2}\left(\mathbf{C}^{n}, d \mu\right),
$$

where

$$
K(x, y)=e^{\langle x, y\rangle / 2} .
$$

For a bounded linear operator $T$ on $A^{2}\left(\mathbf{C}^{n}, d \mu\right)$, the Berezin transform of $T$ is the function $\widetilde{T}$ on $\mathbf{C}^{n}$ defined by

$$
\widetilde{T}(z)=\left\langle T k_{z}, k_{z}\right\rangle
$$

where

$$
k_{z}(x):=\frac{K(x, z)}{K(z, z)^{1 / 2}}
$$

is the normalized reproducing kernel at $z$, i.e. the unit vector in $A^{2}\left(\mathbf{C}^{n}, d \mu\right)$ "in the direction of $K(\cdot, z)$ ".

Received by the editors December 23, 2004 and, in revised form, March 1, 2005.

2000 Mathematics Subject Classification. Primary 47B32; Secondary 32A36, 32M15.

Key words and phrases. Bergman kernel, Berezin transform, bounded symmetric domain, invariant differential operator.

The research of the first author was supported by GA AV ČR grant no. A1019304.

The research of the second author was supported by the Swedish Science Council (VR). 
Recently, Coburn [Cob] has shown that $\widetilde{T}$ is always a Lipschitz function, with the Lipschitz constant bounded by a multiple of the norm of $T$. Namely, for any $x, y \in \mathbf{C}^{n}$,

$$
|\widetilde{T}(x)-\widetilde{T}(y)| \leq \sqrt{2}\|T\||x-y| .
$$

Furthermore, this estimate remains in force also for the similarly defined Berezin transform on the usual Bergman space $A^{2}(\Omega, d v)$ of all holomorphic functions in $L^{2}(\Omega, d v)$, for an arbitrary bounded domain $\Omega \subset \mathbf{C}^{n}$, only with $|x-y|$ replaced by the distance $\beta(x, y)$ of $x$ and $y$ with respect to the Bergman metric. For $\Omega=\mathbf{D}$, the unit disc in $\mathbf{C}$, and $\Omega=\mathbf{B}^{n}$, the unit ball of $\mathbf{C}^{n}$, similar observations were made by Suarez [Sua] and Nam, Zheng and Zhong [NZZ], respectively, with applications to the study of the structure of Toeplitz algebras.

In this note, we want to show that the Berezin transform in fact satisfies a much stronger estimate than the above Lipschitz property. For the Fock space, the corresponding result reads as follows. (We are using the usual conventions concerning multi-indices, i.e. $\partial^{\alpha}$ stands for

$$
\frac{\partial^{|\alpha|}}{\partial z_{1}^{\alpha_{1}} \ldots \partial z_{n}^{\alpha_{n}}}, \quad \text { where } \frac{\partial}{\partial z_{j}}=\frac{1}{2}\left(\frac{\partial}{\partial x_{j}}-i \frac{\partial}{\partial y_{j}}\right) \quad \text { for } z_{j}=x_{j}+i y_{j},
$$

and similarly for $\bar{\partial}$.)

Theorem 1. For any bounded linear operator $T$ on $A^{2}\left(\mathbf{C}^{n}, d \mu\right)$, the Berezin transform $\widetilde{T}$ has bounded derivatives of all orders; i.e. for any multi-indices $\alpha, \beta$,

$$
\left\|\partial^{\alpha} \bar{\partial}^{\beta} \widetilde{T}\right\|_{\infty} \leq c_{\alpha \beta}\|T\|
$$

for some constants $c_{\alpha \beta}$ depending only on $\alpha, \beta$ and $n$.

This theorem is not actually new, but is mentioned without proof in [Cob] as a (probably unpublished) result of Jingbo Xia.

Furthermore, we also have the analogous, and much more interesting, assertion for $\mathbf{C}^{n}$ replaced by a bounded symmetric domain.

Theorem 2. Let $\Omega=\mathcal{G} / \mathcal{K}$ be a bounded symmetric domain in $\mathbf{C}^{n}$ in its HarishChandra realization, with $\mathcal{G}$ the group of all biholomorphic self-maps of $\Omega$ and $\mathcal{K}$ the stabilizer in $\mathcal{G}$ of the origin $0 \in \Omega$. For a bounded linear operator $T$ on $A^{2}(\Omega, d v)$, let $\widetilde{T}^{\#}$ denote the function on $\mathcal{G}$ defined by

$$
\widetilde{T}^{\#}(g):=\widetilde{T}(g 0), \quad g \in \mathcal{G} .
$$

Then, for any left-invariant differential operator $L$ on $\mathcal{G}$,

$$
\left\|L \widetilde{T}^{\#}\right\|_{\infty} \leq c_{L}\|T\|
$$

for some constant $c_{L}$ depending only on $L$ and $\Omega$.

Note that the "invariant derivatives" $L \widetilde{T}^{\#}$ of $\widetilde{T}$ in the last theorem occur, for instance, also in the definition of the Schwarz space on a bounded symmetric domain; see e.g. GaVa, Corollary 6.1.12. The algebra $\left\{f: L f^{\#} \in L^{\infty}\right.$ for any left-invariant differential operator $L$ on $\mathcal{G}\}$, where $f^{\#}(g):=f(g 0)$, occurred also in the recent paper Eng, and was denoted there by $I B C^{\infty}(\Omega)$ (supposed to mean "invariant $B C^{\infty}$ ", i.e. the invariant version of the space $B C^{\infty}$ of all $C^{\infty}$ functions whose partial derivatives of all orders are bounded); thus the last theorem says 
that for any bounded linear operator $T$ in $A^{2}(\Omega, d v)$, with $\Omega$ a bounded symmetric domain, the Berezin transform $\widetilde{T}$ belongs to $I B C^{\infty}(\Omega)$.

The proofs of Theorem 1 and Theorem 2 are given in Section 3, after reviewing some preliminaries in Section 2. Then Sections 4 and 5 discuss further generalizations of these theorems; in particular, we show that they are both a special case of a more general result concerning Bergman spaces with respect to any measure quasi-invariant with respect to a transitive action of a Lie group, and point out a further extension to more general "operator-symbol" assignments than the Berezin transform.

It would be interesting to know whether the above results can also be extended, like the original Lipschitzness result of Coburn, to the context of any bounded domain $\Omega$ in $\mathbf{C}^{n}$ equipped with the Bergman metric. More specifically, if $\nabla$ stands for the covariant differentiation with respect to the Bergman metric, and $\|\cdot\|_{z}$ for the local norm of a tensor at a point $z \in \Omega$, is it true that for each $k=0,1,2, \ldots$. and bounded linear operator $T$ on $A^{2}(\Omega, d v)$, the Berezin transform $\widetilde{T}$ of $T$ satisfies

$$
\sup _{z \in \Omega}\left\|\nabla^{k} \widetilde{T}\right\|_{z} \leq c_{k}\|T\|
$$

for some constants $c_{k}$ depending only on $k$ and $\Omega$ ?

\section{Preliminaries}

Consider, quite generally, an arbitrary domain $\Omega \subset \mathbf{C}^{n}$, a (nonnegative, regular, Borel) measure $d \nu$ on $\Omega$, and let $A^{2}(\Omega, d \nu)$ be the subspace of all holomorphic functions in $L^{2}(\Omega, d \nu)$. Under mild hypothesis on $\nu$ - for instance, whenever it has a positive continuous density with respect to the Lebesgue measure - this subspace is closed and the evaluation functionals $f \mapsto f(z)$, for any $z \in \Omega$, are continuous on it; hence, there exists a function $K(x, y)$ on $\Omega \times \Omega$, called the reproducing kernel, with the property that

$$
\begin{aligned}
& K(\cdot, y) \in A^{2}(\Omega, d \nu) \quad \forall y \in \Omega, \text { and } \\
& f(y)=\int_{\Omega} f(x) K(y, x) d \nu(x) \quad \forall f \in A^{2}(\Omega, d \nu) .
\end{aligned}
$$

The function $K(x, y)$ is holomorphic in $x$ and $\bar{y}$ (hence, in particular, its restriction $K(x, x)$ to the diagonal is real-analytic), and satisfies $K(x, y)=\overline{K(y, x)}$ and $K(x, x) \geq 0$ for any $x, y \in \Omega$. We will usually assume that $\Omega$ and $\nu$ are such that the last inequality is strict, i.e.

$$
K(x, x)>0 \quad \forall x \in \Omega .
$$

Equivalently, for each $x \in \Omega$ there should exist a function $f \in A^{2}(\Omega, d \nu)$ such that $f(x) \neq 0$. This will be the case, for instance, whenever the measure $\nu$ is finite, since $A^{2}(\Omega, d \nu)$ then contains the constant functions.

If (2.1) holds, then we may consider the normalized reproducing kernels

$$
k_{y}:=\frac{K(\cdot, y)}{\|K(\cdot, y)\|}=\frac{K(\cdot, y)}{K(y, y)^{1 / 2}},
$$

and define the Berezin transform of a bounded linear operator $T$ on $A^{2}(\Omega, d \nu)$ by

$$
\widetilde{T}(z):=\left\langle T k_{z}, k_{z}\right\rangle,
$$

with the inner product being taken in $A^{2}(\Omega, d \nu)$. 
Recall (see e.g. [HP]) that a mapping $f$ from a domain in $\mathbf{R}^{n}$ into a Banach space is said to possess a strong derivative $\frac{\partial f}{\partial x_{1}}(x)$ at a point $x=\left(x_{1}, \ldots, x_{n}\right) \in \mathbf{R}^{n}$ if

$$
\lim _{t \rightarrow 0}\left\|\frac{f\left(x_{1}+t, x_{2}, \ldots, x_{n}\right)-f\left(x_{1}, x_{2}, \ldots, x_{n}\right)}{t}-\frac{\partial f}{\partial x_{1}}\left(x_{1}, \ldots, x_{n}\right)\right\|=0,
$$

and similarly for $\frac{\partial f}{\partial x_{2}}, \ldots, \frac{\partial f}{\partial x_{n}}$ and for higher order derivatives. For $\mathbf{C}^{n}$ in the place of $\mathbf{R}^{n}$, we can similarly define the strong holomorphic and anti-holomorphic derivatives $\partial^{\alpha}$ and $\bar{\partial}^{\alpha}$; and finally, using the local coordinate charts, we can define strong derivatives also for functions defined on real or complex manifolds.

Lemma 3. Let $\Omega$ be a domain in $\mathbf{C}^{n}, \nu$ a measure on $\Omega$ such that $A^{2}(\Omega, d \nu)$ is closed and has a reproducing kernel $K(x, y)$, and $x$ a point of $\Omega$ for which $K(x, x)>0$. Then the function $z \mapsto k_{z}$ from $\Omega$ into $A^{2}(\Omega, d \nu)$ has strong derivatives of all orders at $x$.

Proof. Due to the Leibniz rule, it is enough to prove the assertion for the numerical function $K(z, z)^{-1 / 2}$, which is obvious in view of the real-analyticity of $K(z, z)$, and for the vector-valued function $z \mapsto K(\cdot, z)$ from $\Omega$ into $A^{2}(\Omega, d \nu)$. Let $\rho>0$ be so small that the closed polydisc $\overline{D(x, \rho)}$ with center $x$ and radius $\rho$ lies in $\Omega$. Since $K(\cdot, z)$ is conjugate-holomorphic in $z$, we have for any $y \in D(x, \rho)$ by the Cauchy formula

$$
K(\cdot, y)=\frac{1}{(2 \pi)^{n}} \int_{0}^{2 \pi} \ldots \int_{0}^{2 \pi} \frac{K(\cdot, \zeta)}{\left(\bar{\zeta}_{1}-\bar{y}_{1}\right) \ldots\left(\bar{\zeta}_{n}-\bar{y}_{n}\right)} d \theta_{1} \ldots d \theta_{n}
$$

where $\zeta=\left(x_{1}+\rho e^{i \theta_{1}}, \ldots, x_{n}+\rho e^{i \theta_{n}}\right)$ and the integral converges in norm. Moreover, this formula can be differentiated under the integral sign with respect to $y$ any number of times. As the required assertion obviously holds for the numerical function $y \mapsto 1 /\left(\bar{\zeta}_{1}-\bar{y}_{1}\right) \cdots\left(\bar{\zeta}_{n}-\bar{y}_{n}\right)$, uniformly in $\zeta$ as $y$ ranges in the polydisc $D(x, \rho / 2)$, the claim follows.

Corollary 4. Under the same hypothesis, the trace-class-operator-valued function

$$
z \mapsto\left\langle\cdot, k_{z}\right\rangle k_{z}
$$

from $\Omega$ into the space of trace-class operators on $A^{2}(\Omega, d \nu)$ has strong derivatives of all orders at $x$.

Proof. This is immediate from the last lemma, the Leibniz rule, and the elementary estimate

$$
\begin{aligned}
\|\langle\cdot, a\rangle b-\langle\cdot, c\rangle d\|_{\operatorname{tr}} & \leq\|\langle\cdot, a-c\rangle b\|_{\operatorname{tr}}+\|\langle\cdot, c\rangle(b-d)\|_{\text {tr }} \\
& \leq\|a-c\|\|b\|+\|b-d\|\|c\| .
\end{aligned}
$$

\section{Proofs of Theorems 1 and 2}

Proof of Theorem 1. For brevity, let us denote by $A(z)$ the rank one projection

$$
A(z):=\left\langle\cdot, k_{z}\right\rangle k_{z} .
$$

Thus

$$
\widetilde{T}(z)=\operatorname{tr}(T A(z)) .
$$


Consequently, for any multi-indices $\alpha$ and $\beta$,

$$
\partial^{\alpha} \bar{\partial}^{\beta} \widetilde{T}(z)=\operatorname{tr}\left(T \partial^{\alpha} \bar{\partial}^{\beta} A(z)\right),
$$

the interchange of the differentiation and the trace being justified by the existence of the strong derivative $\partial^{\alpha} \bar{\partial}^{\beta} A(z)$ (guaranteed by Corollary 4 ) and the joint continuity of the bilinear form $\operatorname{tr}(X Y)$ with respect to $\|X\|$ and $\|Y\|_{\text {tr }}$ :

$$
|\operatorname{tr}(X Y)| \leq\|X\|\|Y\|_{\text {tr }}
$$

(see e.g. GK]). From the last inequality we also get

$$
\left|\partial^{\alpha} \bar{\partial}^{\beta} \widetilde{T}(z)\right| \leq\|T\|\left\|\partial^{\alpha} \bar{\partial}^{\beta} A(z)\right\|_{\mathrm{tr}} .
$$

For $a \in \mathbf{C}^{n}$, consider the Weyl operators

$$
W_{a} f(z):=k_{a}(z) f(z-a)
$$

on $A^{2}\left(\mathbf{C}^{n}, d \mu\right)$. It is well known (and easily checked) that $W_{a}$ is unitary, and

$$
W_{a} k_{z}=e^{i \operatorname{Im}\langle z, a\rangle / 2} k_{z+a} .
$$

Hence,

$$
W_{a} A(z) W_{a}^{*}=A(z+a) .
$$

Consequently,

$$
\begin{aligned}
\partial_{z}^{\alpha} \bar{\partial}_{z}^{\beta} A(z+a) & =\partial_{z}^{\alpha} \bar{\partial}_{z}^{\beta}\left[W_{a} A(z) W_{a}^{*}\right] \\
& =W_{a}\left[\partial^{\alpha} \bar{\partial}^{\beta} A(z)\right] W_{a}^{*} .
\end{aligned}
$$

Evaluating at $z=0$ and using translation-invariance of the derivatives, it therefore follows that

$$
\partial^{\alpha} \bar{\partial}^{\beta} A(a)=W_{a}\left[\partial^{\alpha} \bar{\partial}^{\beta} A(0)\right] W_{a}^{*} .
$$

As the trace norm is a unitary invariant, we thus get

$$
\left\|\partial^{\alpha} \bar{\partial}^{\beta} A(a)\right\|_{\mathrm{tr}}=\left\|\partial^{\alpha} \bar{\partial}^{\beta} A(0)\right\|_{\mathrm{tr}} \quad \forall a \in \mathbf{C}^{n},
$$

so

$$
\left\|\partial^{\alpha} \bar{\partial}^{\beta} \widetilde{T}\right\|_{\infty} \leq\|T\|\left\|\partial^{\alpha} \bar{\partial}^{\beta} A(0)\right\|_{\text {tr }}
$$

and the claim follows.

Remark. Using the explicit formula (1.2) for $K(x, y)$, it is not difficult to obtain various bounds for $\left\|\partial^{\alpha} \bar{\partial}^{\beta} A(0)\right\|_{\text {tr. }}$. For instance, let us denote, for the moment, the function $K(\cdot, z)=e^{\langle\cdot, z\rangle / 2}$ by $K_{z}$. By the binomial formula,

$$
\partial^{\alpha} \bar{\partial}^{\beta}\left\langle\cdot, k_{z}\right\rangle k_{z}=\sum_{\substack{\gamma \subset \alpha \\
\delta \subset \beta}}\left(\begin{array}{l}
\alpha \\
\gamma
\end{array}\right)\left(\begin{array}{l}
\beta \\
\delta
\end{array}\right)\left[\partial^{\gamma} \bar{\partial}^{\delta} e^{-\|z\|^{2} / 2}\right]\left\langle\cdot, \bar{\partial}_{z}^{\alpha-\gamma} K_{z}\right\rangle \bar{\partial}_{z}^{\beta-\delta} K_{z} .
$$

Since $e^{-\|z\|^{2} / 2}=\sum_{\alpha} z^{\alpha} \bar{z}^{\alpha} / \alpha !(-2)^{|\alpha|}$, we have

$$
\left.\partial^{\alpha} \bar{\partial}^{\beta} e^{-\|z\|^{2} / 2}\right|_{z=0}=\delta_{\alpha \beta} \frac{\alpha !}{(-2)^{|\alpha|}} .
$$


Also, $\bar{\partial}_{z}^{\alpha} K_{z}(x)=(x / 2)^{\alpha} K_{z}(x)$, so $\left.\left\|\bar{\partial}_{z}^{\alpha} K_{z}\right\|\right|_{z=0}=\left\|(x / 2)^{\alpha}\right\|=\sqrt{\alpha ! / 2^{|\alpha|}}$. Consequently,

$$
\begin{aligned}
\left\|\partial^{\alpha} \bar{\partial}^{\beta} A(0)\right\|_{\operatorname{tr}} & \leq \sum_{\gamma \subset \alpha, \beta}\left(\begin{array}{l}
\alpha \\
\gamma
\end{array}\right)\left(\begin{array}{l}
\beta \\
\gamma
\end{array}\right) \frac{\gamma !}{2^{|\gamma|}} \sqrt{\frac{(\alpha-\gamma) !(\beta-\gamma) !}{2^{|\alpha|+|\beta|-2|\gamma|}}} \\
& =\frac{\alpha ! \beta !}{\sqrt{2^{|\alpha|+|\beta|}}} \sum_{\gamma \subset \alpha, \beta} \frac{1}{\gamma ! \sqrt{(\alpha-\gamma) !(\beta-\gamma) !}} \\
& \leq \frac{\alpha ! \beta !}{\sqrt{2^{|\alpha|+|\beta|}}} \sum_{\gamma} \frac{1}{\gamma !}=\frac{\alpha ! \beta !}{\sqrt{2^{|\alpha|+|\beta|}}} e^{n} .
\end{aligned}
$$

This has obvious implications regarding the membership of $A(z)$ - and, hence, of $\widetilde{T}$ for any bounded linear operator $T$ - in various Gevrey classes, etc.; for instance, we see that the radius of convergence of the Taylor series $\sum_{\alpha, \beta} \frac{z^{\alpha} \bar{z}^{\beta}}{\alpha ! \beta !} \partial^{\alpha} \bar{\partial}^{\beta} \widetilde{T}(x)$, for any $x \in \mathbf{C}^{n}$, is at least $\sqrt{2}$. Clearly, it would be of interest to have an explicit formula for $\left\|\partial^{\alpha} \bar{\partial}^{\beta} A(0)\right\|_{\mathrm{tr}}$, but the computation seems difficult.

Proof of Theorem 2. Now let $\Omega$ be a bounded symmetric domain in $\mathbf{C}^{n}$ in its Harish-Chandra realization (so $\Omega$ is circular with respect to the origin and convex). Recall that the group $\mathcal{G}$ of all biholomorphic self-maps of $\Omega$ is a semi-simple Lie group with finite center, and denoting by $\mathcal{K}$ the stabilizer of the origin $0 \in \Omega$ in $\mathcal{G}$ we may (and will) identify $\Omega$ with the coset space $\mathcal{G} / \mathcal{K}$. Any function $f$ on $\Omega$ can thus be lifted to a function $f^{\#}$ on $\mathcal{G}$ by composing with the canonical projection $\mathcal{G} \rightarrow \mathcal{G} / \mathcal{K}=\Omega$, i.e.

$$
f^{\#}(g):=f(g 0), \quad g \in \mathcal{G}, g 0 \in \Omega .
$$

A differential operator $L$ on $\mathcal{G}$ is called left-invariant if

$$
L(f \circ g)=(L f) \circ g \quad \forall g \in \mathcal{G},
$$

where, for a function $f$ on $\mathcal{G}, f \circ g$ stands for the translated function $g_{1} \mapsto f\left(g g_{1}\right)$.

For $g \in \mathcal{G}$, denote by $\mathrm{Jac}_{g}$ the complex Jacobian of the mapping $g: \Omega \rightarrow \Omega$. The change-of-variable formula shows that the operators

$$
U_{g} f(z):=\operatorname{Jac}_{g^{-1}}(z) f\left(g^{-1} z\right)
$$

are isometries on $L^{2}(\Omega, d v)$ and $A^{2}(\Omega, d v)$. Further, $U_{g_{1}} U_{g_{2}}=U_{g_{1} g_{2}}$, so that the operators $U_{g}$ are in fact unitary and $g \mapsto U_{g}$ is a unitary representation of $\mathcal{G}$ on $A^{2}(\Omega, d v)$. Since

$$
\begin{aligned}
\left\langle f, U_{g} k_{z}\right\rangle & =\left\langle U_{g^{-1}} f, k_{z}\right\rangle=K(z, z)^{-1 / 2}\left(U_{g^{-1}} f\right)(z) \\
& =K(z, z)^{-1 / 2} \operatorname{Jac}_{g}(z) f(g z) \\
& =\left\langle f, K(z, z)^{-1 / 2} \overline{\operatorname{Jac}_{g}(z)} K(g z, g z)^{1 / 2} k_{g z}\right\rangle,
\end{aligned}
$$

for any $f \in A^{2}(\Omega, d v)$, we see that $U_{g} k_{z}=\epsilon_{g, z} k_{g z}$, where $\epsilon_{g, z}$ is the constant $K(g z, g z)^{1 / 2} K(z, z)^{-1 / 2} \overline{\operatorname{Jac}_{g}(z)}$. Since $U_{g}$ is unitary and both $k_{z}$ and $k_{g z}$ are unit vectors, we must have $\left|\epsilon_{g, z}\right|=1$. Using again the notation $A(z)=\left\langle\cdot, k_{z}\right\rangle k_{z}$, we thus have

$$
U_{g} A(z) U_{g}^{*}=A(g z) \quad \forall z \in \Omega, g \in \mathcal{G}
$$


Now let $L$ be a left-invariant differential operator on $\mathcal{G}$. From the relation

$$
\widetilde{T}^{\#}(g)=\operatorname{tr}(T A(g 0))
$$

we get as before, denoting $A^{\#}(g):=A(g 0)$,

$$
\left|L \widetilde{T}^{\#}(g)\right| \leq\|T\|\left\|L A^{\#}(g)\right\|_{\mathrm{tr}},
$$

where the $L$ on the right-hand side is understood in the strong sense (its existence being guaranteed by Corollary 4). Now by (3.1) and (3.2) $(e$ stands for the identity element of $\mathcal{G}$ ),

$$
\begin{aligned}
L A^{\#}(g) & =\left[L A^{\#}(\cdot)\right](g e) \\
& =\left[L\left(A^{\#}(g \cdot)\right)\right](e) \\
& =\left[L\left(U_{g} A^{\#}(\cdot) U_{g}^{*}\right)\right](e) \\
& =U_{g}\left[L A^{\#}(e)\right] U_{g}^{*},
\end{aligned}
$$

so again $\left\|L A^{\#}(g)\right\|_{\mathrm{tr}}=\left\|L A^{\#}(e)\right\|_{\mathrm{tr}}$ and

$$
\left\|L \widetilde{T}^{\#}\right\|_{\infty} \leq\|T\|\left\|L A^{\#}(e)\right\|_{\text {tr }},
$$

which gives the required assertion, with $c_{L}=\left\|L A^{\#}(e)\right\|_{\mathrm{tr}}$. This completes the proof of Theorem 2

\section{QUASI-INVARIANT MEASURES}

Both Theorem 1 and Theorem 2 are special cases of the following more general result. Consider, quite generally, an arbitrary domain $\Omega \subset \mathbf{C}^{n}$, and let $\mathcal{G}$ be a Lie group acting on $\Omega$ transitively (i.e. for any $x, y \in \Omega$, there is $g \in \mathcal{G}$ with $g x=y$ ) by biholomorphic transformations. A measure $d \mu$ on $\Omega$ is called quasi-invariant if for each $g \in \mathcal{G}$

$$
\frac{d \mu(g z)}{d \mu(z)}=\left|j_{g}(z)\right|^{2}
$$

for some holomorphic function $j_{g}$ on $\Omega$.

Theorem 5. Let $\mathcal{G}$ be a Lie group acting transitively on $\Omega$ by holomorphic transformations, and $d \mu$ a quasi-invariant measure on $\Omega$. Assume that the Bergman space $A^{2}(\Omega, d \mu)$ has a reproducing kernel $K(x, y)$ and $K(z, z)>0 \forall z \in \Omega$, so that the normalized reproducing kernels $k_{z}$ and, hence, the Berezin transform $T \mapsto \widetilde{T}(z)=$ $\left\langle T k_{z}, k_{z}\right\rangle$ are defined. Fix a basepoint $z_{0} \in \Omega$ and let $\widetilde{T}^{\#}$ be the function on $\mathcal{G}$ defined by

$$
\widetilde{T}^{\#}(g):=\widetilde{T}\left(g z_{0}\right), \quad g \in \mathcal{G} .
$$

Then for any left-invariant differential operator $L$ on $\mathcal{G}$,

$$
\left\|L \widetilde{T}^{\#}\right\|_{\infty} \leq c_{L}\|T\| \quad \forall T \in \mathcal{B}\left(A^{2}(\Omega, d \mu)\right),
$$

for some constant $c_{L}$ depending only on $L, \Omega$ and $d \mu$.

It is clear that the Lebesgue measure is quasi-invariant, under any $\mathcal{G}$, since (4.1) holds with $j_{g}=\mathrm{Jac}_{g}$. Thus Theorem 2 indeed follows from Theorem 5 upon taking for $\mathcal{G}$ the group of all biholomorphic self-maps of $\Omega$ and $z_{0}=0$. Similarly, the Gaussian measure (1.1) on $\mathbf{C}^{n}$ is quasi-invariant with respect to translations, since for $g(z)=z-c, c \in \mathbf{C}^{n}$, (4.1) clearly holds with

$$
j_{g}(z)=e^{\langle z, c\rangle / 2-\|c\|^{2} / 4} .
$$


Thus Theorem 1 follows from Theorem 5 upon taking $\mathcal{G}=\left\{z \mapsto z-c: c \in \mathbf{C}^{n}\right\}$ and $z_{0}=0$. (Note that translation-invariant differential operators on $\mathbf{C}^{n}$ are precisely the differential operators with constant coefficients, and $\partial^{\alpha} \bar{\partial}^{\beta}$, where $\alpha, \beta$ range over all multi-indices, serve as a basis for the latter.) Some other applications of Theorem 5 are given below.

Proof of Theorem 5. For $g \in \mathcal{G}$, let $U_{g}$ be the operator

$$
U_{g} f(z):=j_{g^{-1}}(z) f\left(g^{-1} z\right)
$$

The "change of variable" formula (4.1) then again shows that $U_{g}$ is an isometry on $L^{2}(\Omega, d \mu)$ and $A^{2}(\Omega, d \mu)$. Furthermore, it follows from (4.1) that

$$
\left|j_{g_{2}}\left(g_{1} z\right) \cdot j_{g_{1}}(z)\right|=\left|j_{g_{1} g_{2}}(z)\right| \quad \forall z \in \Omega, g_{1}, g_{2} \in \mathcal{G}
$$

which implies that $\left(j_{g_{2}} \circ g_{1}\right) \cdot j_{g_{1}}=\epsilon\left(g_{1}, g_{2}\right) j_{g_{1} g_{2}}$ for some unimodular constant $\epsilon\left(g_{1}, g_{2}\right)$; consequently,

$$
U_{g_{1}} U_{g_{2}}=\epsilon\left(g_{2}^{-1}, g_{1}^{-1}\right) U_{g_{1} g_{2}}
$$

Taking $g_{2}=g_{1}^{-1}$, it follows that the operators $U_{g}$ are invertible, with $U_{g}^{-1}=$ $\overline{\epsilon\left(g, g^{-1}\right)} U_{g^{-1}}$. Thus the operators $U_{g}$ are in fact unitary, and $g \mapsto U_{g}$ is a projective unitary representation of $\mathcal{G}$ on $A^{2}(\Omega, d \mu)$. It follows as in the proof of Theorem 2 that $U_{g} k_{z}=\epsilon_{g, z} k_{g z}$, where $\epsilon_{g, z}=K(z, z)^{-1 / 2} \epsilon\left(g, g^{-1}\right) \overline{j_{g}(z)} K(g z, g z)^{1 / 2}$, and $\left|\epsilon_{g, z}\right|=1$. Using again the notation $A(z)=\left\langle\cdot, k_{z}\right\rangle k_{z}$, we thus have as before

$$
U_{g} A(z) U_{g}^{*}=A(g z) \quad \forall z \in \Omega, g \in \mathcal{G}
$$

and the rest of the proof is the same as for Theorem 2 ,

As an application on Theorem 5, recall that the Bergman kernel $K(x, y)$ of any domain $\Omega$ (with respect to the Lebesgue measure) enjoys the transformation property

$$
K(x, y)=K(g z, g y) \cdot \operatorname{Jac}_{g}(x) \cdot \overline{\operatorname{Jac}_{g}(y)}
$$

for any biholomorphic self-map $g$ of $\Omega$. Consequently, if $K(z, z)>0 \forall z$ (for instance, if $\Omega$ is bounded), then for any $\alpha \in \mathbf{R}$ the measure

$$
d \mu_{\alpha}(z):=K(z, z)^{-\alpha} d v(z)
$$

on $\Omega$ is quasi-invariant with respect to any group $\mathcal{G}$ of holomorphic self-maps of $\Omega$, with $j_{g}=\operatorname{Jac}_{g}^{1+\alpha}$. Furthermore, it is easy to see that the corresponding weighted Bergman spaces $A^{2}\left(\Omega, d \mu_{\alpha}\right)$ are nontrivial and contain the constant functions as soon as $\alpha$ is not too small ( $\alpha \geq 0$ will do). By Theorem 5 , we thus have the following.

Corollary 6. Let $\Omega$ be any bounded domain on which a group $\mathcal{G}$ of its biholomorphic self-maps acts transitively. Then for any $\alpha \geq 0$, the conclusion of Theorem 2 holds for the Berezin transform $T \mapsto \widetilde{T}$ on the space $A^{2}\left(\Omega, d \mu_{\alpha}\right)$.

In particular, it follows that Theorem 2 generalizes to the standard families $A^{2}\left(\Omega, d \mu_{\alpha}\right)$ of weighted Bergman spaces on any bounded homogeneous (not necessarily symmetric) domains $\Omega$ in $\mathbf{C}^{n}$.

Remark. As another application of Theorem 5. it is easy to see that Theorem 1 remains in force also for the Segal-Bargmann-Fock spaces $A^{2}\left(\mathbf{C}^{n}, d \mu_{\alpha}\right)$ with respect to the scaled Gaussians

$$
d \mu_{\alpha}(z):=\left(\frac{\alpha}{\pi}\right)^{n} e^{-\alpha\|z\|^{2}} d v(z)
$$


for any $\alpha>0$. However, this can also be easily gleaned from the previous case $\alpha=\frac{1}{2}$ by the change of variable $z \mapsto \sqrt{2 \alpha} z$.

\section{Symbolid CALCULi}

We conclude by discussing briefly an extension of Theorems 15 to more general assignments $T \mapsto \widetilde{T}$ than the Berezin transforms. Namely, consider again the situation from Theorem 5 i.e. a Lie group $\mathcal{G}$ acting on $\Omega$ transitively by holomorphic transformations and a quasi-invariant measure $d \mu$ on $\Omega$. Let $U_{g}$ be the unitary operators (4.2), $z_{0}$ a fixed basepoint in $\Omega$, and denote by $\mathcal{K}$ the stabilizer of $z_{0}$ in $\mathcal{G}$. Let $X$ be any trace class operator on $A^{2}(\Omega, d \mu)$ such that

$$
X U_{k}=U_{k} X \quad \forall k \in \mathcal{K} .
$$

For $z \in \Omega$, set

$$
A(z):=U_{g} X U_{g}^{*} \quad \text { for any } g \in \mathcal{G} \text { such that } g z_{0}=z .
$$

In view of (5.1), this definition is consistent, i.e. the right-hand side is independent of the choice of $g \in \mathcal{G}$ sending $z_{0}$ into $z$.

We can now define a "generalized Berezin transform" by

$$
\widetilde{T}(z):=\operatorname{tr}(T A(z)) .
$$

Clearly, the ordinary Berezin transform from the previous sections is recovered upon choosing $X=\left\langle\cdot, k_{z_{0}}\right\rangle k_{z_{0}}$.

It is immediate that the whole proof of Theorem 5 works, without any need of modifications, also for these more general Berezin transforms, as long as we have an analogue of Lemma 3 , i.e. as long as the function $A(z)$ has strong derivatives of all orders; in other words, using representation-theoretic terminology, as long as $X$ is a smooth vector for the (not only projective but honest) representation $\rho: g \mapsto U_{g} X U_{g}^{*}$ of $\mathcal{G}$ on $A^{2}(\Omega, d \mu)$. We thus obtain the following generalization of Theorem 5

Theorem 7. Under the same hypotheses as in Theorem 5 , let $X$ be any trace class operator on $A^{2}(\Omega, d \mu)$ which satisfies (5.1) and is a smooth vector for $\rho$. Then the conclusion of Theorem 5 remains in force also for the associated generalized Berezin transform (5.2).

The condition that $X$ be a smooth vector for $\rho$ is as a rule satisfied if $X$ is "fabricated" from the reproducing kernels in some way; in particular, in view of Lemma 3, it is fulfilled whenever $X$ is a linear combination of rank-one operators of the form $\left\langle\cdot, h_{\alpha}\right\rangle h_{\beta}$, where for a multi-index $\alpha, h_{\alpha}=\left.\bar{\partial}_{z}^{\alpha} K(\cdot, z)\right|_{z=0}$.

In the context of weighted Bergman spaces (and their analytic continuations) on bounded symmetric domains, transforms of the form (5.2) were investigated by Arazy and Upmeier $\mathrm{AU}$ ] under the name of invariant symbolic calculi. (More precisely, the symbolic calculi are the adjoint maps, assigning to a function $f$ on $\Omega$ the operator

$$
T_{f}:=\int_{\Omega} f(\xi) A(\xi) d \iota(\xi)
$$

on $A^{2}(\Omega, d \mu)$, where $d \iota(z)=K(z, z) d v(z)$ is the $\mathcal{G}$-invariant measure on $\Omega$.) 
Examples of such calculi- for which $X$ is moreover a smooth vector for $\rho$, in view of the comment two paragraphs above - include, in particular, the $m$-Berezin transforms defined in Suarez [Sua] and Nam, Zheng and Zhong [NZZ]. In particular, Theorem 7 gives a strengthening of Theorem 2.11 in [NZZ].

\section{REFERENCES}

[AU] J. Arazy, H. Upmeier: Invariant symbolic calculi and eigenvalues of invariant operators on symmetric domains, Function spaces, interpolation theory, and related topics (Lund, 2000) (A. Kufner, M. Cwikel, M. Engliš, L.-E. Persson, and G. Sparr, editors), pp. 151211, Walter de Gruyter, Berlin, 2002. MR 1943284 (2003k:32031)

[Cob] L.A. Coburn: A Lipschitz estimate for Berezin's operator calculus, Proc. Amer. Math. Soc. 133 (2005), 127-131. MR2085161 (2005e:47060)

[Eng] M. Engliš: Berezin-Toeplitz quantization on the Schwartz space of bounded symmetric domains, J. Lie Theory 15 (2005), 27-50. MR2115226

[GaVa] R. Gangolli, V.S. Varadarajan, Harmonic analysis of spherical functions on real reductive groups, Springer-Verlag, Berlin-Heidelberg, 1988. MR0954385 (89m:22015)

[GK] I.C. Gohberg, M.G. Krein, Introduction to the theory of linear nonselfadjoint operators, Translations of Mathematical Monographs 18, Amer. Math. Soc., Providence, 1969. MR0246142 (39:7447)

[HP] E. Hille, R.S. Phillips, Functional analysis and semi-groups, American Mathematical Society Colloquium Publications 31, Amer. Math. Soc., Providence, 1957. MR0089373 (19:664d)

[NZZ] K. Nam, D. Zheng, C. Zhong: m-Berezin transform and compact operators, preprint (2004), submitted to Rev. Mat. Iberoamer.

[Sua] D. Suarez: Approximation and symbolic calculus for Toeplitz algebras on the Bergman space, Rev. Mat. Iberoamer. 20 (2004), 563-610. MR2073132 (2005e:47075)

Mathematics Institute, Academy of Sciences of the Czech Republic, Žitná 25, 11567 Praha 1, CZech Republic

E-mail address: englis@math.cas.cz

Chalmers Tekniska Högskola/Göteborgs Universitet, 41296 Göteborg, Sweden

E-mail address: genkai@math.chalmers.se 